\title{
Mapping Periodic Lattice Distortions in Exfoliated Dichalchogenides with Atomic Resolution cryo-STEM
}

Robert Hovden ${ }^{1}$, Adam W. Tsen ${ }^{2}$, Pengzi Liu ${ }^{1}$, Benjamin H. Savitzky ${ }^{4}$, Yu Liu ${ }^{5}$, Wenjian Lu ${ }^{5}$, Yuping Sun $^{5,6,7}$, Philip Kim ${ }^{8}$, Abhay N. Pasupathy ${ }^{3}$, Lena F. Kourkoutis ${ }^{1,9}$

${ }^{1}$ School of Applied and Engineering Physics, Cornell University, Ithaca, NY 14853, USA

${ }^{2}$ Department of Chemistry, University of Waterloo, Waterloo, ON N2L 3G1, Canada

${ }^{3}$ Department of Physics, Columbia University, New York, New York 10027, USA

${ }^{4}$ Department of Physics, Cornell University, Ithaca, NY 14853, USA

${ }^{5}$ Key Laboratory of Materials Physics, Chinese Academy of Sciences, Hefei 230031, China

${ }^{6}$ High Magnetic Field Laboratory, Chinese Academy of Sciences, Hefei 230031, China

${ }^{7}$ Centre of Advanced Microstructures, Nanjing University, Nanjing 210093, China

${ }^{8}$ Department of Physics, Harvard University, Cambridge, Massachusetts 02138, USA

${ }^{9}$ Kavli Institute at Cornell for Nanoscale Science, Ithaca, New York 14853, USA

Charge density waves (CDW) and their concomitant periodic lattice distortions (PLD) govern the electronic properties in many layered transition-metal dichalcogenides [1,2]. In particular, $1 \mathrm{~T}-\mathrm{TaS}_{2}$ can undergo a PLD phase transition from a conducting to an insulating state as the PLD becomes commensurate with the crystal lattice $[3,4]$. Here we directly image PLDs of the nearly-commensurate (NC) and commensurate (C) phases in thin exfoliated $1 \mathrm{~T}-\mathrm{TaS}_{2}$ using atomic resolution cryogenic scanning transmission electron microscopy (cryo-STEM). At low temperatures, we observe commensurate PLD superstructures. Figure 1 shows atomic-resolution HAADF cryo-STEM imaging of the commensurate periodic lattice displacements (PLD) at low temperature (95K) in thin-flake 1T$\mathrm{TaS}_{2}$ - here, bright atoms represent the heavy Ta sites. The PLD creates a visible low-frequency contrast modulation with periodicity of $\sim 1.2 \mathrm{~nm}$. Overlays in Fig. 1 highlight a unit cell in the commensurate PLD of a $\sim 65$ layer flake. The visibility of this PLD in projection implies at least partial stacking order of the PLDs. For disordered stacking, the supercell will be lost when viewed in projection.

Using aberration corrected HAADF-STEM, stacking domains and their boundaries were observed in exfoliated $\mathrm{NC} 1 \mathrm{~T}-\mathrm{TaS}_{2}$. In Fig. 2a, 1T-TaS 2 appears as bright Ta atoms in a trigonal array. This is the expected stacking for the perfect $1 \mathrm{~T}$ polymorph, which we denote as A..A.. (Fig. 2c). However, A..B.. stacking domains with a hexagonal array of Ta atoms was also present in the same flake (Fig. 2b). More importantly, we show that PLDs exist in domains with and without stacking faults in the atomic lattice and their boundaries. The observed one-bond length transition (Fig. 2d) in stacking order is expected to create a fractional shift in the CDW between adjacent layers that should affect interlayer coupling and the electronic properties of $1 \mathrm{~T}-\mathrm{TaS}_{2}$, especially in thin samples. Real-space characterization of the local topology and PLD structure across the phase diagram by cryo-STEM will enable harnessing of emergent properties of thin transition-metal dichalcogenides.

[1] Wilson, J. A., Di Salvo, F. J. \& Mahajan, S., Advances in Physics 24, 117-201 (1975).

[2] Tsen, A. W., Hovden, R., Wang, D. Z., Kim, Y. D., Okamoto, J., Spoth, K. A., Liu, Y., Lu, W. J., Sun, Y. P., Hone, J., Kourkoutis, L. F., Kim, P. \& Pasupathy, A. N. Proc. Natl. Acad. Sci. U.S.A.

112, 15054- 15059 (2015)

[3] Thompson, A. H., Gamble, R. F. \& Revelli, J. F., Solid State Communications 9, 981-985 (1971).

[4] Ishiguro, T. \& Sato, H., Phys. Rev. B 44, 2046-2060 (1991).

[5] The work at Cornell is supported by the Packard Foundation and made use of the Cornell Center for Materials Research Shared Facilities supported through the NSF MRSEC program (DMR-1120296). 

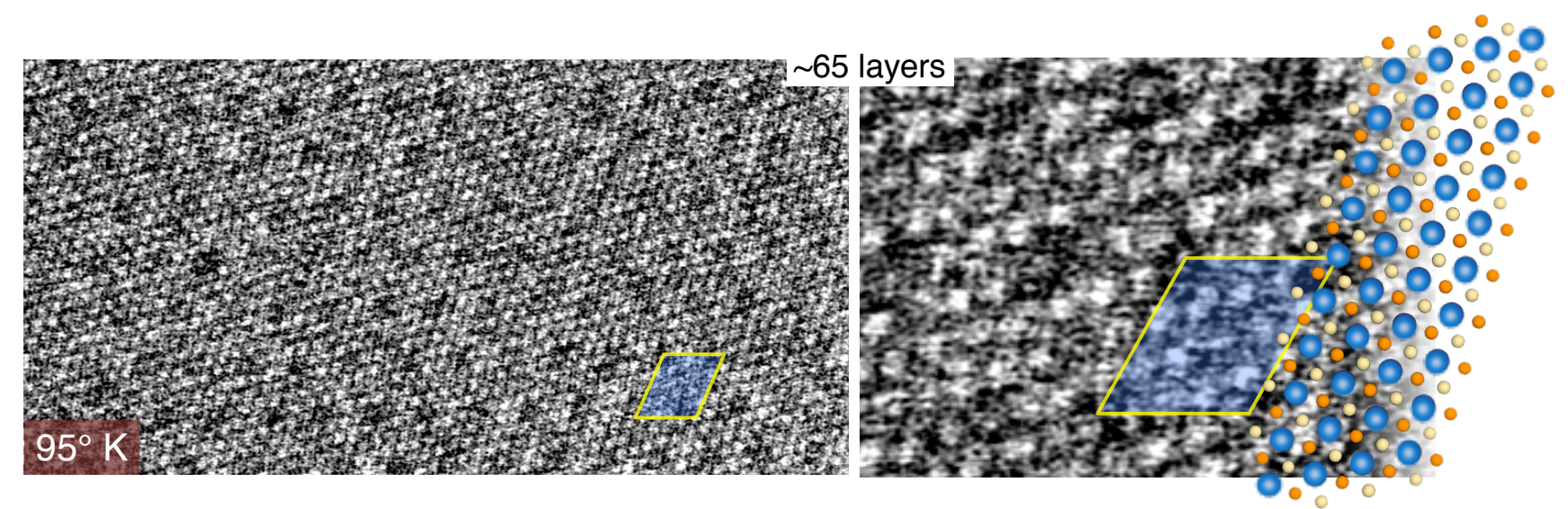

Figure $1 \mid$ PLD ordering and atomic resolution HAADF-STEM imaging of thin C phase $1 \mathrm{~T}-\mathrm{TaS}_{2}$. At $95 \mathrm{~K}$ the C-PLD structure is markedly visible in HAADF images of thicker exfoliated flakes. This suggests at least partial ordering of the PDLs in the out-of-plane direction. The PLD repeat structure is highlighted by a blue parallelogram with $1.2 \mathrm{~nm}$ sides.
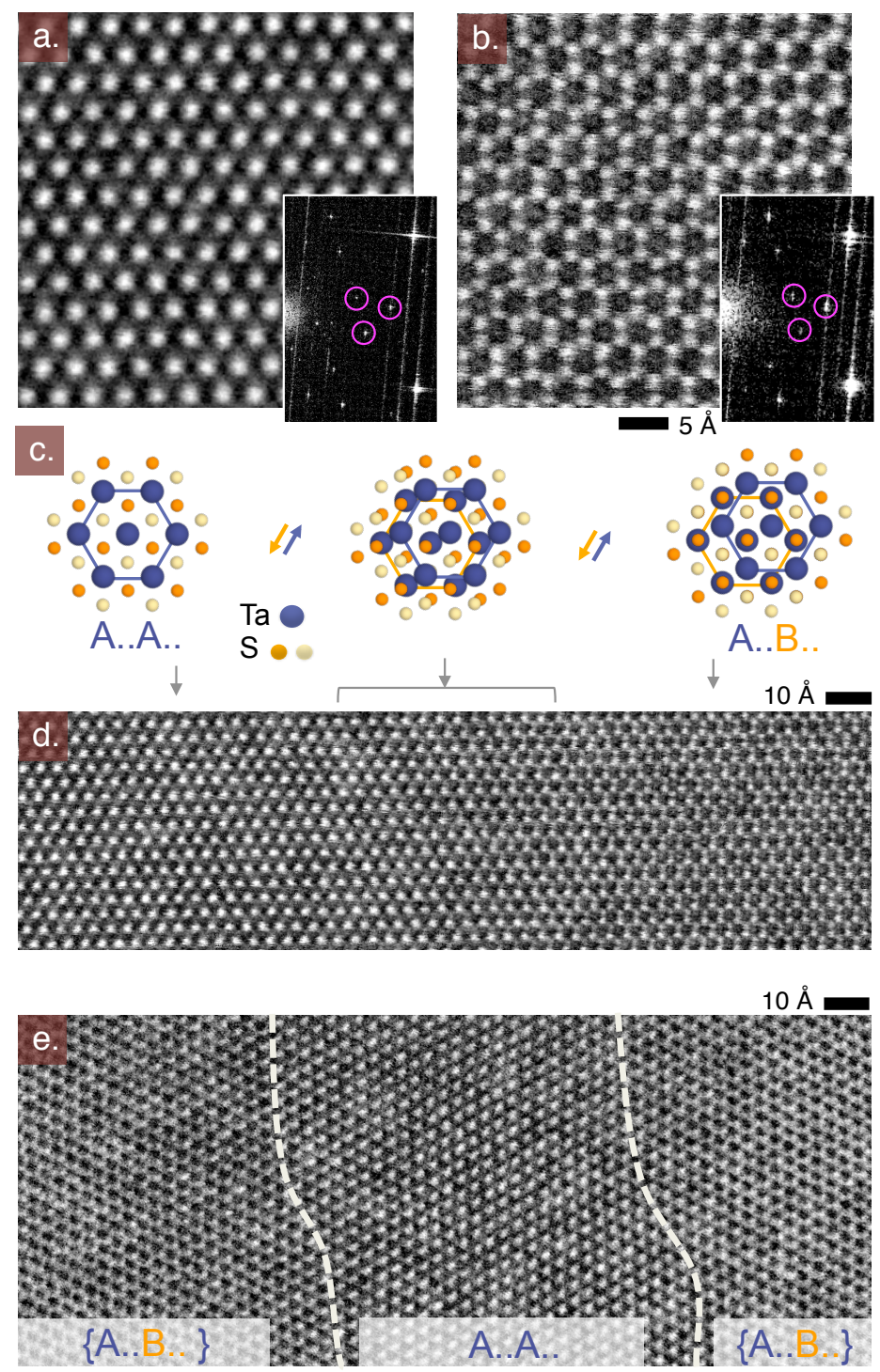

Figure 2 Atomic resolution imaging of stacking order domain boundaries in thin $1 \mathrm{~T}-\mathrm{TaS}_{2}$. Bright atoms represent $\mathrm{Ta}$ in these HAADF-STEM images. (a) Often observed, a trigonal stacking (denoted A..A..) arrangement in which all Ta atoms lie atop each other when viewed along the c-axis (planar vector). (b) Hexagonal stacking (denoted A..B..) was also observed with roughly half of the layers having Ta sites shifted one bond length relative to the other layers. Insets $(a, b)$ show the Fourier transform from each specimen region with PLD peaks present in both stacking orders. c) An illustration shows the stacking arrangements as they transition from A..A.. to A..B.. d) The domain boundary between the two stacking orders was observed experimentally, transitioning continuously over $\sim 30 \AA$ from trigonal (left) to hexagonal (right) stacking. e) A domain boundary that transitions from $\{$ A..B.. $\}$ to A..A.. and back to $\{$ A..B.. $\}$ over $\sim 15 \mathrm{~nm}$; braces denote either A..B.. or B..A.. stacking. 